\title{
Comparison of the Efficacy of Definitive Radiotherapy and Radical Prostatectomy in High-Risk Prostate Cancer: A Single-Center Experience
}

\author{
(•) Berrin İnanç ${ }^{1}$, (0) Özlem Mermut ${ }^{1}$, ๑ Uğur Yücetaş ${ }^{2}$ \\ 1 University of Health Sciences Turkey, Istanbul Training and Research Hospital, Clinic of Radiation Oncology, Istanbul, Turkey \\ 2University of Health Sciences Turkey, Istanbul Training and Research Hospital, Clinic of Urology, Istanbul, Turkey
}

\begin{abstract}
Objective: This retrospective study aimed to examine treatment outcomes and patient selection criteria in individuals with high-risk prostate cancer (PCa) treated with definitive radiotherapy (RT) and androgen deprivation therapy (ADT) or radical prostatectomy (RP).

Materials and Methods: In total, 72 patients treated with definitive RT or RP for high-risk PCa between 2011 and 2018 were included in the study. Patient characteristics, treatment data, and follow-up data were obtained from the patient's file.

Results: Of 72 patients with high-risk PCa, 34 (46.6\%) received definitive RT and ADT and 38 (52.1\%) had undergone RP. The median follow-up time in the RP group was 44.5 (range, 14-100) months and that in the RP group was 48 (range, 9-108) months. No significant between-group difference was found in the biochemical recurrence-free survival, metastasis-free survival, and overall survival (OS) rates after 3 and 5 years of follow-ups ( $\geq 00.005$ ). In a subgroup analysis, RT was the treatment of choice for patients aged $\geq 65$ years and for those with prostate-specific antigen values of $\geq 20 \mathrm{ng} / \mathrm{dL}$, a Gleason score (GS) of 9-10, and T stage T3-4 and $\mathrm{N}+$ status $(\mathrm{p}=0.015,0.001,0.035$, and 0.022 , respectively). In the univariate and multivariate analyses, age $\geq 65$ years and GS of 8-10 were significant risk factors for reduced OS in all high-risk PCa cases.

Conclusion: No significant difference was found in the survival outcome of patients in the RT + ADT and RP groups. RT should be preferred in patients aged $\geq 65$ years and in those with a high T stage and GS of 8-10.
\end{abstract}

Keywords: Prostate cancer, radical prostatectomy, radiotherapy

\section{Introduction}

Prostate cancer (PCa) is the second most common cancer in men and the fifth most common cause of death among men worldwide (1). It has one of the highest mortality rates among all cancers, despite surgery and radiotherapy (RT), with a high rate of relapse and progression, especially in those with high-risk PCa (2). Historically, RT, androgen deprivation therapy (ADT), or a combination of both was the standard treatment for patients with high-risk PCa. Several studies have now suggested that a radical prostatectomy (RP) can control disease progression and improve survival $(3,4,5,6)$. However, there is no consensus on the optimal treatment for patients with high-risk localized PCa [ $\leq \mathrm{T} 2 \mathrm{c}$ or a Gleason score (GS) of 8-10 or prostate-specific antigen (PSA) level of $>20 \mathrm{ng} / \mathrm{dL}$ ]. Current guidelines recommend both RT and RP for high-risk PCa (7).
The advantages and disadvantages of both regimens should be considered in treatment decision making. While RP guides the selection of patients who can benefit from adjuvant therapies by correct staging, it cannot eradicate micrometastatic diseases (8). By contrast, a combination of RP and ADT eliminates pelvic micrometastases in high-risk cases. However, the long treatment period (i.e., 6-8 weeks) is a major disadvantage of RT + ADT.

Regarding patient selection for RT and RP, the side effect profiles of each treatment regimen must be considered. The side effects in patients with high-risk PCa who underwent RP frequently included impotence, urinary incontinence, and bleeding, whereas those in patients who received RT commonly included bladder and bowel complaints $(9,10)$. These RT-related side effects were more common after irradiation using conventional RT techniques, such as conformal techniques, rather than new methods (e.g., image-guided RT and volumetric arc therapy).

Cite this article as: İnanç B, Mermut Ö, Yücetaş U. Comparison of the Efficacy of Definitive Radiotherapy and Radical Prostatectomy in High-Risk Prostate Cancer: A Single-Center Experience. Bull Urooncol 2021;20(4):225-230

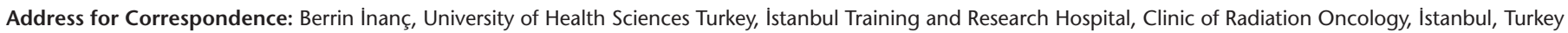
Phone: +90 2124596692 E-mail: byalcin77@hotmail.com ORCID-ID: orcid.org/0000-0002-6354-4609 Received: 15.02.2021 Accepted: 06.05.2021 
In patients with high-risk $\mathrm{PCa}$, all treatment decisions regarding the use of RT + ADT or RP should be based on long-term survival data, tumor control rates, and treatment side effects, as well as patient preference.

In this study, we examined treatment outcomes and patient selection criteria in individuals with high-risk PCa treated by definitive RT or radical RP.

\section{Materials and Methods}

\section{Patient Characteristics}

In this retrospective study, we evaluated 72 patients diagnosed with high-risk PCa who were treated with RT + ADT or RP in İstanbul Training and Research Hospital, Department of Radiation Oncology, between January 2011 and December 2018. All patients underwent pelvic computed tomography (CT) or pelvic magnetic resonance imaging in addition to a bone scan. Some patients underwent prostate-specific membrane antigen positron emission tomography (PSMA PET/CT) to exclude distant metastasis.

The inclusion criteria were as follows: a histologically proven adenocarcinoma of the prostate gland, high-risk PCa according to D'Amico's risk classification criteria ( $\geq T 2 c$ or a GS of 8-10 or PSA level of $>20 \mathrm{ng} / \mathrm{dL}$ ), treatment with RP or RT + ADT, and data were available including survival outcomes. Patients who had distant metastases and evidence of clinical pelvic lymph node involvement were excluded. All relevant laboratory and pathology results were obtained from the hospital's database. Data related to the treatment follow-up were obtained from clinical files.

The study was approved by the local ethics committee of our hospital. All patients were given a thorough explanation of the study, and informed consent was obtained from all of them (approval no: 2021-2664).

\section{RT- and Surgery-Related Data}

In the definitive RT group, intensity-modulated radiotherapy (IMRT) or volumetric-modulated arc therapy (VMAT) was applied. The treatment schedule was as follows: external beam RT in 1.8-2.0 Gy daily fractions with $6 \mathrm{MV}$ photon beams at 5 days a week. A total dose of 46 Gy was delivered to the pelvic region, 54 Gy to the seminal vesicle, and 76-78 Gy to the prostate. According to the risk stratification based on Partin's tables (11), the entire pelvic region was included in the RT field in patients whose pelvic lymph node involvement risk exceeded $15 \%$. The gross tumor volume included the prostate volume. The clinical target volume was defined as follows: CTV1 comprised the prostate only, CTV2 comprised CTV1 plus the seminal vesicles, and CTV3 comprised CTV1 plus CTV2 plus pelvic lymph nodes. The planning treatment volume was defined as pelvic lymph nodes with $0.7 \mathrm{~mm}$ margin. CTV2 and CTV1 were defined as 8 $\mathrm{mm}$ in all directions and $5 \mathrm{~mm}$ in the posterior direction.

Postoperative RT was given as adjuvant or salvage treatment in the RP group. Adjuvant RT was administered 4-6 months after surgery. Most of the patients in the RP group had positive surgical margins. Salvage RT was applied in the event of biochemical failure. In the RP group, biochemical failure was defined as an increase in the PSA level of $>0.1 \mathrm{ng} / \mathrm{mL}$ postoperatively. In the RT group, it was defined according to the Phoenix criteria as a PSA level of $2 \mathrm{ng} / \mathrm{mL}$ above the lowest level (12). Postoperative RT was applied to the operating bed with daily fractions of 1.82.0 Gy with $6 \mathrm{MV}$ photon beams. Surgical treatment comprised $\mathrm{RP}$ and pelvic lymph node dissection.

\section{Outcomes and Follow-up}

The biochemical recurrence-free survival (BRFS), metastasis-free survival (MFS), and overall survival (OS) rates of the patients in each treatment group were recorded. BRFS, MFS, and OS were defined as the time from RP/RT until biochemical failure, metastasis, and death from any cause, respectively.

Treatment toxicity was evaluated using the Common Terminology Criteria for Adverse Events version 4.0 (13). During RT, all patients were assessed at least once a week. At this time, they underwent a clinical examination, and their blood count was measured. After RT, their PSA levels were checked every 3 months in the first 2 years, and abdominal/pelvic tomography and bone scanning were performed every 6 months. Followup was performed every 6 months for 2-5 years and once a year after 5 years. During the follow-up period, all patients with suspected local or regional recurrence and distant metastasis were referred for PSMA PET/CT and multiparametric magnetic resonance imaging.

\section{Statistical Analyses}

The compliance of the variables to normal distribution was examined using histogram graphics and the KolmogorovSmirnov test. The mean, standard deviation, and median values were used while presenting descriptive analyzes. Categorical variables were compared using the Pearson chi-square test. The Mann-Whitney $U$ test was used in evaluating nonparametric variables between two groups. BRFS, MFS, and OS rates were evaluated using Kaplan-Meier analysis. The multivariate Cox proportional hazard model was used to evaluate interactions between two groups and prognostic variables for OS outcomes. All analyses were performed at a 95\% confidence level with a 0.05 significance level using SPSS for Windows version 17.0 (SPSS Inc., Chicago, IL, USA).

\section{Results}

This study included 72 patients with high-risk PCa: 34 (46.6\%) patients in the definitive RT group and 38 (52.1\%) patients in the RP group. Table 1 provides information on the baseline parameters of the patients and their treatment. The mean age of those in the RP and RT groups was 63.8 (57-76) and 66.7 (51-78) years, respectively, with a significant between-group difference $(p=0.045)$. The proportion of patients with a mean age $\geq 65$ years with a pretreatment PSA level, PSA $>20 \mathrm{ng} /$ $\mathrm{dL}$, and last PSA level was higher in the RT group $(\mathrm{p}=0.015$, $p=0.001, p=0.001$, and $p=0.006$, respectively). Moreover, the GS in the RT group was significantly higher than that in the RP group ( $<8$ vs 8 and $9-10, p=0.035$ ). Regarding the National Comprehensive Cancer Network (NCCN) risk classification, RP was the treatment of choice in 15 (44.5\%) patients in the highrisk group, whereas RT was preferred in 31 (86.5\%) patients in 

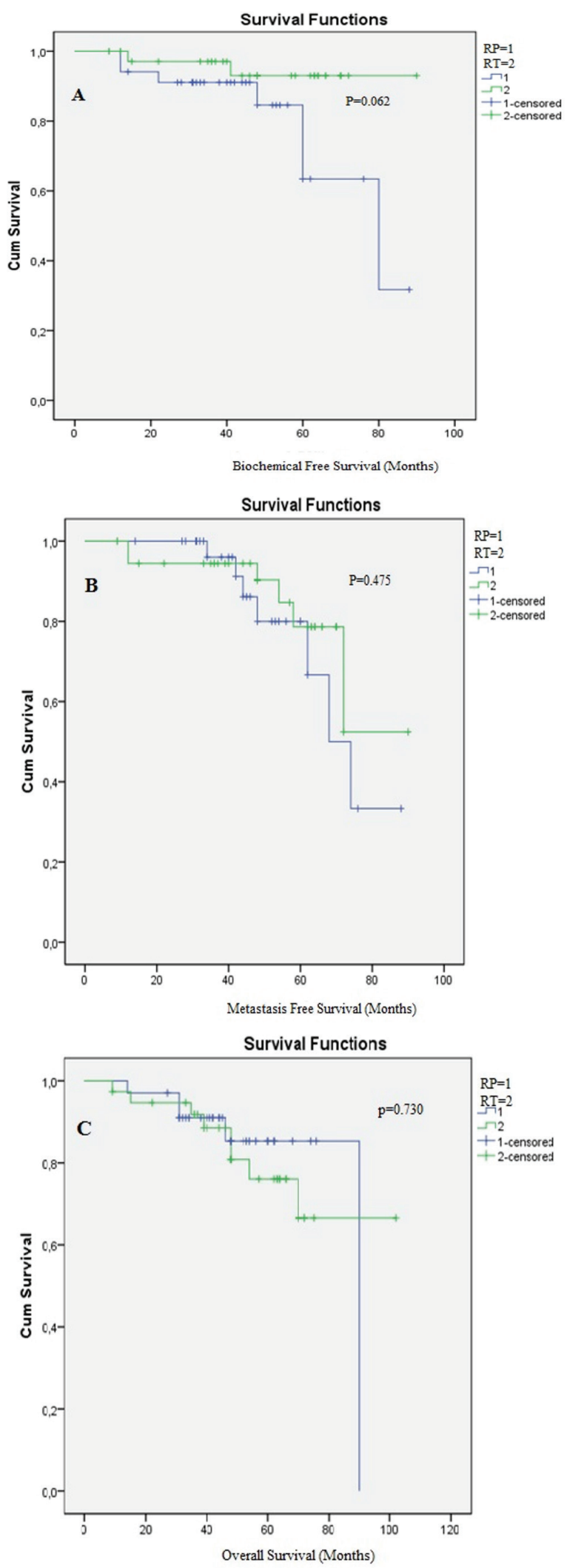

Figure 1. Kaplan-Meier curve for BFS, MFS and OS (A, B, C)

OS: Overall survival, BFS: Biochemical recurrence-free survival, MFS: Metastasisfree survival the very high-risk group (Gleason pattern 5 and/or $>4$ score with GS 8-10, $\mathrm{p}=0.018$ ). According to the $7^{\text {th }}$ American Joint Committee on Cancer Tumor-Node-Metastasis staging system, 33 (82.3\%) patients were stage T2 (T2c) and 20 (99.5\%) were stage T3 and T4 in the RP group, and $11(100 \%)$ were $\mathrm{N}(+)$ in the RT group. RT was the treatment of choice for patients with advanced disease stages, showing a significant difference $(\mathrm{p}=0.001)$. The follow-up time was 44.5 (range, 14-100) months in the RP group and 48 (range, 9-108) months in the RT group $(\mathrm{p}=0.230)$.

Moreover, 16 (47\%) patients received adjuvant RT and $6(15.7 \%)$ patients received salvage RT due to biochemical recurrence in the RP group. In the RT group, ADT (50 mg of bicalutamide and $22.5 \mathrm{mg}$ of leuprolide acetate) was given to all patients who received RT for 3 years. Neoadjuvant ADT was given to six patients before RT because of the tumor size. Only two patients did not receive ADT because of severe heart failure.

The Kaplan-Meier curves for BRFS, MFS, and OS times are shown in Figure 1. No significant difference was found in the BRFS, MFS,

Table 1. Comparison of patients characteristics in treatmen groups

\begin{tabular}{|c|c|c|c|}
\hline & $\begin{array}{l}\text { Radical } \\
\text { prostatectomy }\end{array}$ & $\begin{array}{l}\text { Definitive } \\
\text { radiotherapy }\end{array}$ & p-value \\
\hline \multicolumn{4}{|l|}{ Age } \\
\hline Mean, (range) & $63.82 \pm 4.2(57-76)$ & $66.79 \pm 7.6(51-78)$ & 0.045 \\
\hline$<65$ years & $24(60 \%)$ & $16(40 \%)$ & 0.015 \\
\hline$\geq 65$ years & $10(31.3 \%)$ & $22(68.8 \%)$ & \\
\hline $\begin{array}{l}\text { Pretreatment } \\
\text { PSA value } \\
\text { mean (range) }\end{array}$ & $9.7 \pm 14.5(3.4-66)$ & $25.50 \pm 34.10(4.6-146)$ & 0.001 \\
\hline
\end{tabular}

First PSA

\begin{tabular}{|l|l|l|l|}
\hline$<10 \mathrm{ng} / \mathrm{dL}$ & $18(72 \%)$ & $7(28 \%)$ & 0.001 \\
\hline $10-20 \mathrm{ng} / \mathrm{dL}$ & $10(52.6 \%)$ & $9(27.4 \%)$ & \\
\hline$\geq 20 \mathrm{ng} / \mathrm{dL}$ & $6(21.4 \%)$ & $22(78.6 \%)$ & \\
\hline \multicolumn{4}{|l|}{ Gleason score } \\
\hline$<8$ & $15(44.1 \%)$ & $7(18.4 \%)$ & 0.035 \\
\hline 8 & $8(25.8 \%)$ & $18(47.5 \%)$ & \\
\hline $9-10$ & $11(32.4 \%)$ & $13(34.2 \%)$ & \\
\hline
\end{tabular}

NCCN risk classification

\begin{tabular}{|c|c|c|c|}
\hline High & $15(44.5 \%)$ & $7(18.5 \%)$ & 0.018 \\
\hline Very high & $19(55.5 \%)$ & $31(85.5 \%)$ & \\
\hline \multicolumn{4}{|c|}{ Clinical stage (n) } \\
\hline $\mathrm{T} 2$ & $33(82.5 \%)$ & $7(17.5 \%)$ & 0.001 \\
\hline T3-4 & $1(0.5 \%)$ & $20(99.5 \%)$ & \\
\hline $\mathrm{N}+$ & 0 & $11(100 \%)$ & \\
\hline $\begin{array}{l}\text { Last PSA, } \\
\text { mean (range) }\end{array}$ & $0.37(0.08-10.4)$ & $7.3(0.08-149)$ & 0.006 \\
\hline $\begin{array}{l}\text { Follow-up } \\
\text { (months) }\end{array}$ & $44.5 \pm 16.7(14-100)$ & $48 \pm 20.1(9-108)$ & 0.230 \\
\hline Alive & $29(85.3 \%)$ & $30(78.9 \%)$ & 0.433 \\
\hline Exitus & $5(14.7 \%)$ & $8(21.1 \%)$ & \\
\hline
\end{tabular}

PSA: Prostate-specific antigen, NCCN: National Comprehensive Cancer Network 
and OS values between the two groups at 3- and 5-year followups (Table 2). The BRFS time in the RP group was longer than that in the RT group ( $85.7 \pm 3.7$ vs $72.4 \pm 5.0$ months, $p=0.062)$, with the difference close to statistical significance.

Table 3 shows the results of the Cox regression analysis of the OS in the two groups. In the univariate analysis, age $(p=0.035)$ and a higher GS (i.e., $\geq 8$ ) were significant predictors of OS ( $p=0.025)$. In the multivariate analysis, age and a higher GS (i.e., $\geq 8$ ) were independent predictive factors for OS in both groups $(p=0.043$ and $p=0.027$, respectively).

\section{Discussion}

In this study, we examined patient outcomes and selection criteria in individuals with high-risk PCa treated with definitive RT or RP. The aim was to shed light on the suitability of different patients for various treatments. We found no difference in the BFS, MFS, and OS of the patients between the two groups, although the BRFS in the RP group was marginally better than that in the RT group.

\begin{tabular}{|c|c|c|c|}
\hline Survival outcomes & $\begin{array}{l}\text { Radical } \\
\text { prostatectomy }\end{array}$ & $\begin{array}{l}\text { Definitive } \\
\text { radiotherapy }\end{array}$ & p-value \\
\hline \multicolumn{4}{|l|}{ BFS } \\
\hline Median \pm SD (month) & $85.7 \pm 3.7$ & $72.4 \pm 5.0$ & \\
\hline 3-year BFS (\%), (95\%, Cl) & $93(83.2-102.8)$ & $84.6(81.5-91.3)$ & 0.062 \\
\hline 5 -year BFS (\%), $(95 \%, \mathrm{Cl})$ & $78.1(67.3-88.9)$ & $63.4(60.6-66.2)$ & \\
\hline \multicolumn{4}{|l|}{ MFS (n) } \\
\hline Median \pm SD (month) & $75.4 \pm 6.8$ & $74 \pm 8.9$ & \\
\hline 3-year MFS (\%), (95\%, Cl) & $90.8(82.7-98.9)$ & $90.3(83.1-97.5)$ & 0.674 \\
\hline 5 -year MFS (\%), $(95 \%, \mathrm{Cl})$ & $70(65.6-74.4)$ & $78.6(73-84.2)$ & \\
\hline \multicolumn{4}{|l|}{ OS } \\
\hline Median \pm SD (month) & $92 \pm 4.3$ & $91 \pm 5.5$ & 0.730 \\
\hline 3-year OS (\%), (95\%, Cl) & $88(76.4-99.6)$ & $89.5(79.9-99.1)$ & \\
\hline 5-year OS (\%), $(95 \%, \mathrm{Cl})$ & $85.2(71.1-99.3)$ & $76.4(69-83.8)$ & \\
\hline
\end{tabular}

Currently, both RT and RP are recommended as first-line treatments for clinically high-risk PCa cases. However, the optimal treatment has not been established. Many studies have attempted to shed light on this issue in recent years $(14,15,16,17)$. Two recent meta-analyses included studies on treatment outcomes of patients with high-risk PCa $(18,19)$. However, the majority of the studies enrolled in these metaanalyses did not include subgroup analyses according to the T-stage, GS, or RT type. Therefore, no comprehensive data can aid clinicians in treatment selection for patients with high-risk PCa.

$\mathrm{RP}$ is generally the treatment of choice in young patients $(<65$ years) with high-performance status and no comorbidities. Before RP, all patients, particularly younger ones, should be informed about potential surgery-related side effects, which include urine leakage and sexual dysfunction. These side effects can cause psychological problems posttreatment in young patients. In the present study, RP was the treatment of choice for those aged $<65$ years $(p=0.015)$. Age was a prognostic factor for OS in the univariate and multivariate analyses $(p=0.035$ and $\mathrm{p}=0.043$, respectively).

A meta-analysis published in 2020 (8) included 25 studies that compared the efficacy of RP and RT in high-risk PCa cases. In the two groups, OS, cancer-specific survival (CSS), BRFS, MFS, and clinical recurrence-free survival were investigated, with detailed subgroup analyses. This meta-analysis revealed that the survival times of the patients who underwent RP were high and that RT delayed disease progression. Based on these findings, the authors concluded that RT should be the primary treatment for patients with a high T-stage or high GS. Similarly, in the present study, RT was the treatment of choice for patients with a high GS $(\geq 8)$ and high T-stage ( $p=0.002$ and $p=0.001$, respectively).

Andic et al. (20) evaluated 120 patients with high-risk PCa who received $R T+\operatorname{ADT}(n=72)$ or RP $(n=40)$. Distant MFS, CSS, and OS were comparable in both groups, but BRFS was significantly lower in the RP group $(p<0.001)$. In the present study, we did find a significant between-group difference in BRFS, MFS, and OS. However, BRFS was longer in the RP group, with the difference close to significance $(p=0.062)$. We attributed this finding to the fact that $16(47 \%)$ of the patients who underwent surgery

Table 3. Predictive factors in the univariate and multivariate analyses for overall survival

\begin{tabular}{|c|c|c|c|c|c|c|}
\hline & \multicolumn{3}{|c|}{ Univariate } & \multicolumn{3}{|c|}{ Multivariate } \\
\hline & OR & $95 \% \mathrm{Cl}$ & p-value & OR & $95 \% \mathrm{Cl}$ & p-value \\
\hline Age (mean) & 1.103 & $1.00-1.208$ & 0.035 & 1.092 & $1.003-1.189$ & 0.043 \\
\hline Pretreatment PSA (ng/dL) & 1.009 & $0.994-1.022$ & 0.251 & - & - & - \\
\hline PSA group (<20 vs $\geq 20$ ) & 0.917 & $0.291-2.892$ & 0.883 & - & - & - \\
\hline \multirow{2}{*}{ Clinical T-stage (T2 vs T3, T4) } & 0.637 & $0.079-5.164$ & 0.673 & - & - & - \\
\hline & 0.478 & $0.052-4.410$ & 0.515 & & & \\
\hline Last PSA (ng/dL) & 0.992 & $1.044-1.178$ & 0.175 & - & - & - \\
\hline Treatment group (RP vs RT) & 1.238 & $1.401-3.820$ & 0.710 & - & - & - \\
\hline
\end{tabular}


also received adjuvant RT. Most of the patients in the RP group required adjuvant RT postoperatively because of positive surgical margins and seminal vesicle involvement. Various studies have reported that RT administered in the early postoperative period after RP reduced PSA levels and improved treatment outcomes $(21,22)$.

In our study, patients with GS of 8-10 were classified in the high-risk group according to the risk classification criteria of the NCCN. Previously, Kishan et al. (23) compared the outcomes of $\mathrm{RT}+$ brachytherapy, RT alone, and RP in patients with GS 9-10 and reported comparable survival times. In the present study, a GS of $>8$ was an independent prognostic factor in both the univariate and multivariate analyses $(p=0.027)$. Moreover, RT was the treatment of choice in very high-risk cases (i.e., a GS of 5).

Several studies have reported that increasing the RT dose in patients with PCa increased BRFS but not OS $(24,25)$. However, these studies have examined outcomes only in patients who received an RT dose of $>70 \mathrm{~Gy}$. In the present study, all patients who received RT received a dose of 76 or 78 Gy because of their high-risk status. Therefore, the RT dose was not investigated in this study.

As a result of the rapid developments in RT techniques, high-dose RT can currently be applied to high-risk PCa cases. Many studies $(26,27)$ have compared RP with RT applied with conventional RT techniques. In recent years, many new RT applications, such as image-guided RT and volumetric-modulated arc therapy, have become available. These techniques can deliver high-dose RT to tumor tissues while protecting healthy tissues. Long-term treatment outcomes, including survival analyses, of patients treated with the latest RT techniques and RP are needed to improve patient selection.

In this study, RT + ADT was the treatment of choice for very highrisk cases. A marginal improvement in BRFS was found in the RP group. The possible reason for this was that approximately half of the patients in this group received postoperative adjuvant RT. In making treatment decisions, both RT and surgery should be offered to patients simultaneously.

\section{Study Limitations}

This study had some limitations. First, patients' quality of life after RT or RP was not assessed. Second, although brachytherapy is recommended after RT in the treatment of high-risk $\mathrm{PCa}$ in the current guidelines (i.e., NCCN), it was not applied in our study because it was not performed in our hospital. Finally, it was necessary to define high-risk PCa cases as high or very highrisk cases. Thus, evaluating treatment outcomes in separate risk categories (i.e., high or very high risk) may be more effective in determining the optimal treatment strategy.

\section{Conclusion}

According to the results of this study and literature findings, treatment outcomes, including survival times, are comparable in high-risk PCa treated with RT or RP. Based on the subgroup analyses, RT should be the treatment of choice for patients with a high $T$ stage and a high GS and aged $\geq 65$ years.

\section{Acknowledgements}

Publication: The results of the study were not published in full or in part in form of abstracts.

Contribution: There is not any contributors who may not be listed as authors.

Conflict of Interest: No conflict of interest was declared by the authors.

Financial Disclosure: The authors declared that this study received no financial support.

\section{Ethics}

Ethics Committee Approval: The study was approved by the local ethics committee of our hospital. All patients were given a thorough explanation of the study (approval no: 2021-2664).

Informed Consent: Informed consent was obtained from all of them.

Peer-review: Externally and internally peer-reviewed.

Authorship Contributions

Supervision: U.Y., Critical Review: U.Y., Concept: B.I.., U.Y., Design: U.Y., Data Collection or Processing: Ö.M., Analysis or Interpretation: B.I., Literature Search: Ö.M., Writing: B.İ.

\section{References}

1. Bray F, Ferlay J, Soerjomataram I, et al. Global cancer statistics 2018: GLOBOCAN estimates of incidence and mortality worldwide for 36 cancers in 185 countries. CA Cancer J Clin 2018;68:394-424.

2. Kohler BA, Sherman RL, Howlader $\mathrm{N}$, et al. Annual report to the Nation on The Status of Cancer, 1975-2011, featuring incidence of breast cancer subtypes by race/ethnicity, poverty, and state. J Natl Cancer Inst 2015;107:djv048. doi: 10.1093/jnci/djv048.

3. Johnstone PA, Ward KC, Goodman M, et al. Radical prostatectomy for clinical T4 prostate cancer. Cancer 2006;106:2603-2609.

4. Spahn M, Weiss $C$, Bader $P$, et al. Longterm outcome of patients with highrisk prostate cancer following radical prostatectomy and stage-dependent adjuvant androgen deprivation. Urol Int 2010;84:164-173.

5. Steuber T, Budäus L, Walz J, et al. Radical prostatectomy improves progression-free and cancer-specific survival in men with lymph node positive prostate cancer in the prostate-specific antigen era: a confirmatory study. BJU Int 2011;107:1755-1761.

6. Siddiqui SA, Boorjian SA, Blute ML, et al. Impact of adjuvant androgen deprivation therapy after radical prostatectomy on the survival of patients with pathological T3b prostate cancer. BJU Int 2011;107:383-388.

7. Mohler JL, Antonarakis ES, Armstrong AJ, et al. Prostate cancer, version 2.2020, NCCN Clinical Practice Guidelines in Oncology. J Natl Compr Canc Netw 2019;17:479-505.

8. Wang $Z, N i$ Y, Chen J, et al. The efficacy and safety of radical prostatectomy and radiotherapy in high-risk prostate cancer: a systematic review and meta-analysis. World J Surg Oncol 2020;18:42.

9. Litwin MS, Gore JL, Kwan L, et al. Quality of life after surgery, external beam irradiation, or brachytherapy for early-s tage prostate cancer. Cancer 2007; 109:2239-2247.

10. Potosky AL, Davis WW, Hoffman RM, et al. Five-year outcomes after prostatectomy or radiotherapy for prostate cancer: the prostate cancer outcomes study. J Natl Cancer Inst 2004;96:1358-1367.

11. Partin AW, Mangold LA, Lamm DM, et al. Contemporary update of prostate cancer staging nomograms (Partin Tables) for the new millennium. Urology 2001;58:843-848. 
12. Roach $M$, Hanks $G$, Thames $H$ Jr, et al. Defining biochemical failure following radiotherapy with or without hormonal therapy in men with clinically localized prostate cancer: recommendations of the RTOG-ASTRO Phoenix consensus conference. Int J Radiat Oncol Biol Phys 2006;65:965-974.

13. US Department of Health and Human Services. Common Terminology Criteria for Adverse Events(CTCAE). Last Accessed Date:14.06.2010. Available from: http://evs.nci.nih.gov/ftp1/ CTCAE/CTCAE_4.03_2010-06-14_QuickReference_8.5x11.pdf

14. Giorgio A, Lidia S, Stefano A, et al. Retrospective comparison of external beam radiotherapy and radical prostatectomy in highrisk, clinically localized prostate cancer. Int J Radiat Oncol Biol Phys 2009; 75:975-982.

15. Chen L, Li Q, Wang $Y$, et al. Comparison on efficacy of radical prostatectomy versus external beam radiotherapy for the treatment of localized prostate cancer. Oncotarget 2017;8:79854-79863.

16. Boorjian SA, Karnes RJ, Viterbo R, et al. Long-term survival after radical prostatectomy versus external-beam radiotherapy for patients with high-risk prostate cancer. Cancer 2011;117:2883-2891.

17. Arcangeli G, Strigari L, Arcangeli $S$, et al. Retrospective comparison of external beam radiotherapy and radical prostatectomy in highrisk, clinically localized prostate cancer. Int J Radiat Oncol Biol Phys 2009;75:975-982.

18. Petrelli F, Vavassori I, Coinu A, et al. Radical prostatectomy or radiotherapy in high-risk prostate cancer: a systematic review and metaanalysis. Clin Genitourin Cancer 2014;12:215-224.

19. Lei JH, Liu LR, Qiang W, et al. Systematic review and meta-analysis of the survival outcomes of first-line treatment options in high-risk prostate cancer. Sci Rep 2015;5:7713.
20. Andic F, Izol V, Gokcay S, et al. Definitive external-beam radiotherapy versus radical prostatectomy in clinically localized high-risk prostate cancer: a retrospective study. BMC Urol 2019;19:3.

21. ThompsonIM, Tangen CM, Paradelo J et al. Adjuvant radiotherapy for pathological T3NOMO prostate cancer significantly reduces risk of metastases and improves survival: long- term follow up of a randomized clinical trial. J Urol 2009,181:956-962.

22. Bolla $M$, van Poppel $H$, Collette $L$, et al. Postoperative radiotherapy after radical prostatectomy: a randomised controlled trial (EORTC trial 22911). Lancet 2005;366:572-578.

23. Kishan AU, Cook RR, Ciezki JP, et al. Radical prostatectomy, external beam radiotherapy, or external beam radiotherapy with brachytherapy boost and disease progression and mortality in patients with gleason score 9-10 prostate cancer. JAMA 2018;319:896-905.

24. Beckendorf V, Guerif S, Le Prisé E, et al. 70 Gy versus 80 Gy in localized prostate cancer: 5-year results of GETUG 06 randomized trial. Int J Radiat Oncol Biol Phys 2011;80:1056-1063.

25. Michalski JM, Moughan J, Purdy J, et al. Effect of standard vs doseescalated radiation therapy for patients with intermediate-risk prostate cancer: The NRG oncology RTOG 0126 randomized clinical trial. JAMA Oncol 2018;4:e180039. doi: 10.1001/jamaoncol.2018.0039.

26. Rosenthal SA, Sandler HM. Treatment strategies for high-risk locally advanced prostate cancer. Nat Rev Urol 2010;7:31-38.

27. Juloori A, Shah C, Stephans K, et al. Evolving paradigm of radiotherapy for high-risk prostate cancer: current consensus and continuing controversies. Prostate Cancer 2016;2016:2420786. doi: $10.1155 / 2016 / 2420786$. 